\title{
CASE REPORT OF A PATIENT PRESENTING WITH METRORRHAGIA DUE TO RETAINED PIECES OF FETAL BONE.
}

Bratati Moitra $^{1}$

\section{HOW TO CITE THIS ARTICLE:}

Bratati Moitra. "Case report of a patient presenting with metrorrhagia due to retained pieces of fetal bone". Journal of Evolution of Medical and Dental Sciences 2013; Vol2, Issue 33, August 19; Page: 6176-6178.

A patient named Saira Bano age - 45 years Wife of - Muhammad Abdullah of Jagdishpur, Champaran, Hazaribagh presented in OPD of RAJENDRA INSTITUTE OF MEDICAL SCIENCES, Ranchi on 07.05.2012 with complain of heavy \& irregular bleeding per vaginum for 6 months. Her Last menstrual period was on 27 $7^{\text {th }}$ April. Her cycles were 7-8 days / $10 \pm 5-7$ days. Bleeding is heavy with passage of clots. Her Obstetrical history - Para $-6+2$, All full term normal delivery at home. Last one was Medical termination of pregnancy of 4 months -6 yrs back by untrained person.

General Examination - Patient was moderately pale. Her pulse was 100/min \& Blood pressure - 124/70 mm of Hg. Chest - Bilateral vesicular breath sound heard \& C.V.S. - Tachycardia was present.

Per Abdominal Examination - Abdomen soft. No tenderness.

Per speculum examination - Cervix unhealthy congested infected discharge present.

Per vaginal Examination - Uterus was retroverted. Slightly bulky Mobility restricted. Fornices free.

Patient was admitted for further investigation. Her Haemoglobin was 8 gm\% Total count \& Differential count of W.B.C, Routine examination of urine, bleeding time \& Clotting time, Blood glucose F \& P/P Blood urea \& Serum Creatinine all were within normal limits. She was HIV and Hepatitis B surface antigen - Negative, Her Chest X ray \& E.C.G. was normal. Patient's Hb was built up by I.V. Iron sucrose + protein diet \& vitamin + micronutrients.

U.S.G. - Report showed Endometrium is thick \& echogenic - looks like old organized missed products.

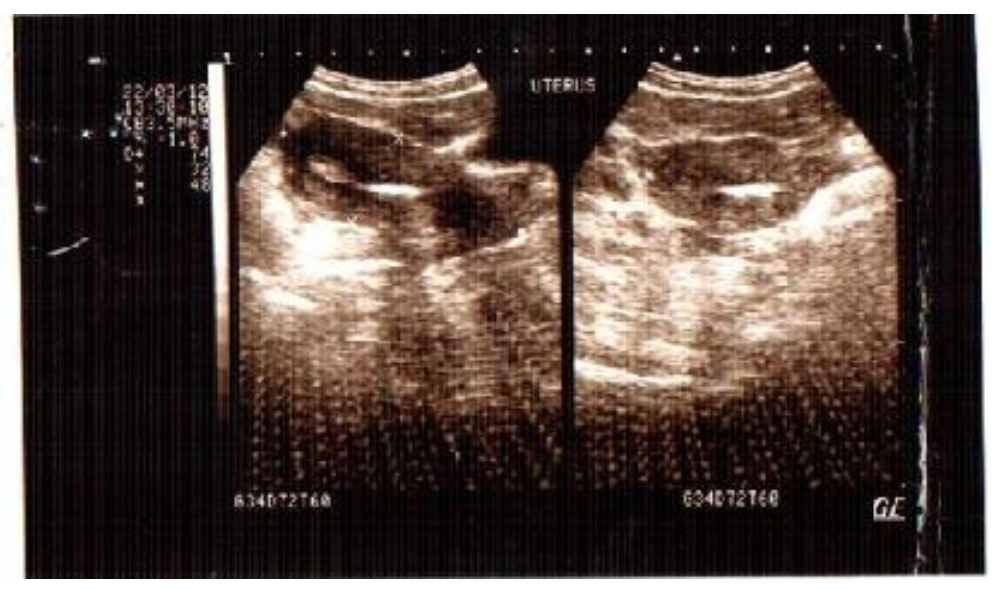


Patient was posted for hysterectomy \& Total Abdominal Hysterectomy with Bilateral Salpingo oophorectomy was done.

After hysterectomy on cutting open the uterine cavity two pieces of fetal bones were found. Bones were flat \& probably scalp bones.

Patient post operative period was un-eventful \& was discharged on $8^{\text {th }}$ post operative day.
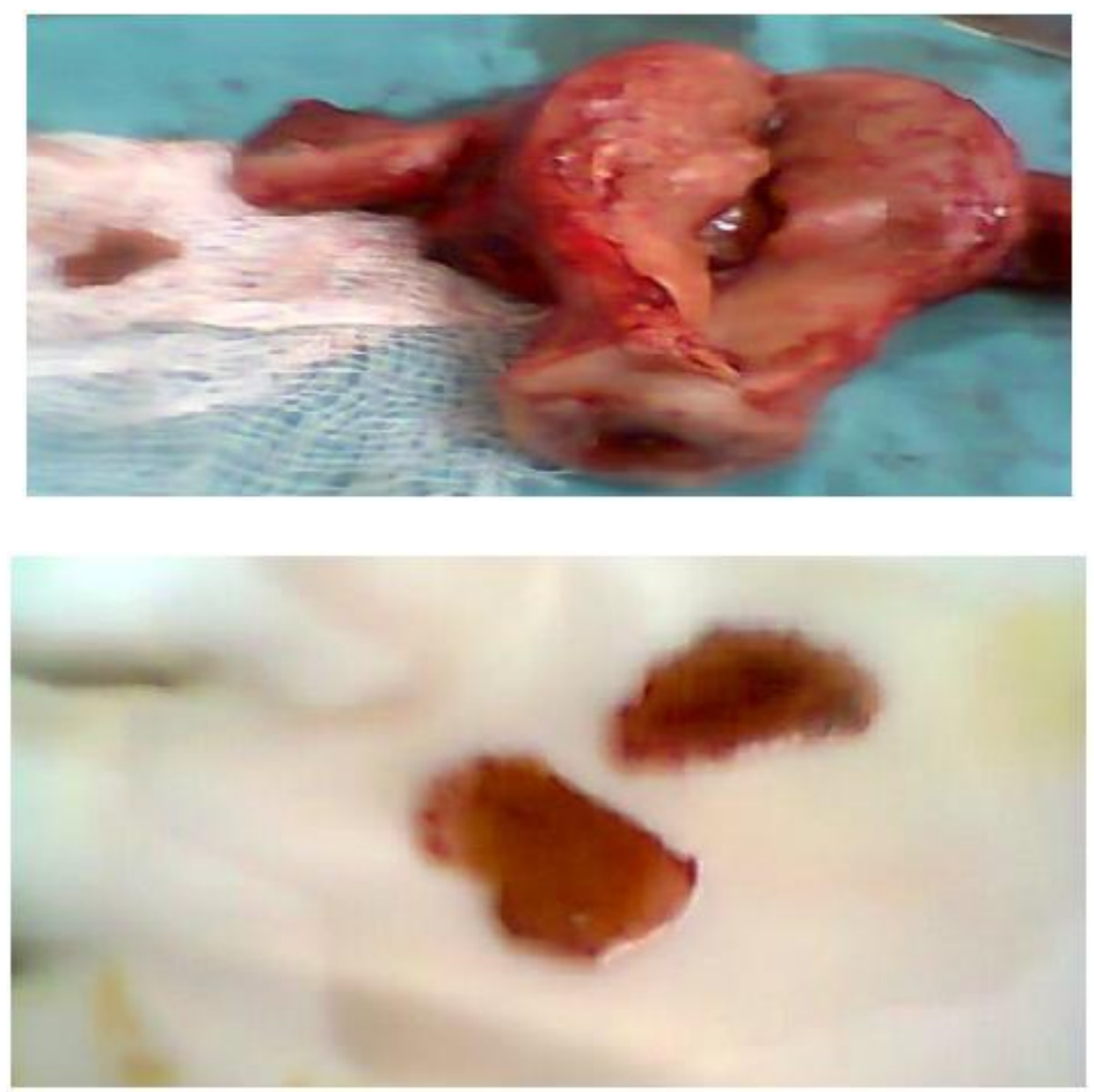

\section{REFERENCES:}

1. Carlso Karen J MD, Eisenstat, Stephonic A MD, Ziporyn, Terra PHD. The new Havard Guide to women health, Havard University.

2. Dubinsky TJ-Value of Sonography in diagnosis of abdominal uterine bleeding J. Clin ultrasound-2004; 32-348-353.

3. Bignardi T, Van Den Bosch T, Condus G- Abdominal uterine \& post menopausal bleeding in the acute Gyanecology unit-Best practice-Clin Obst \& Gynae- 2009;23-595-607. 


\section{CASE REPORT}

\section{AUTHORS:}

1. Bratati Moitra

\section{PARTICULARS OF CONTRIBUTORS:}

1. Assistant Professor, Department of Gynaecology \& Obstetrics, Rajendra Institute of Medical Sciences, Ranchi, Jharkhand.

\section{NAME ADRRESS EMAIL ID OF THE}

\section{CORRESPONDING AUTHOR:}

Dr. Bratati Moitra,

24, Bardwan Compound,

P.O. - Lalpur, Ranchi.

Email - legs2104@yahoo.co.in

Date of Submission: 31/07/2013.

Date of Peer Review: 01/08/2013.

Date of Acceptance: 05/08/2013.

Date of Publishing: 14/08/2013. 